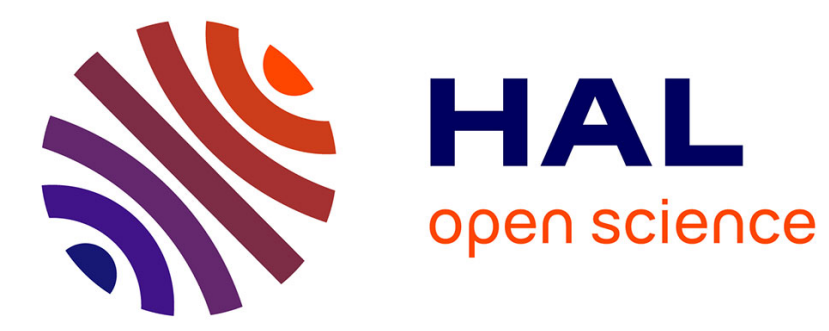

\title{
Urbanization drives an early spring for plants but not for pollinators
}

\author{
Alessandro Fisogni, Nina Hautekèete, Yves Piquot, Marion Brun, Cédric \\ Vanappelghem, Denis Michez, François Massol
}

\section{To cite this version:}

Alessandro Fisogni, Nina Hautekèete, Yves Piquot, Marion Brun, Cédric Vanappelghem, et al.. Urbanization drives an early spring for plants but not for pollinators. Oikos, 2020, 129 (11), pp.1681-1691. 10.1111/oik.07274 . hal-02942280

\section{HAL Id: hal-02942280 \\ https://hal.science/hal-02942280}

Submitted on 6 Nov 2020

HAL is a multi-disciplinary open access archive for the deposit and dissemination of scientific research documents, whether they are published or not. The documents may come from teaching and research institutions in France or abroad, or from public or private research centers.
L'archive ouverte pluridisciplinaire HAL, est destinée au dépôt et à la diffusion de documents scientifiques de niveau recherche, publiés ou non, émanant des établissements d'enseignement et de recherche français ou étrangers, des laboratoires publics ou privés. 
Urbanization drives an early spring for plants but not for pollinators

Alessandro Fisogni ${ }^{1,2}$, Nina Hautekèete ${ }^{1}$, Yves Piquot ${ }^{1}$, Marion Brun ${ }^{4}$, Cédric

Vanappelghem ${ }^{1}$, Denis Michez ${ }^{5}$ and François Massol ${ }^{1,3}$

${ }^{1}$ Univ. Lille, CNRS, UMR 8198 - Evo-Eco-Paleo, FR-59000 Lille, France

${ }^{2}$ Dept of Evolution, Ecology, and Organismal Biology, Univ. of California, Riverside, 900 University Avenue, Riverside, CA 92521, USA

${ }^{3}$ Univ. Lille, CNRS, Inserm, CHU Lille, Inst. Pasteur de Lille, U1019 - UMR 8204 -

CIIL - Center for Infection and Immunity of Lille, Lille, France

${ }^{4}$ Univ. Lille, UFR de Géographie et Aménagement - TVES EA 4477, Lille, France

${ }^{5}$ Laboratoire de Zoologie, Res. Inst. of Biosciences, Univ. of Mons, Mons, Belgium

Corresponding author: Alessandro Fisogni, Univ. Lille, CNRS, UMR 8198 - EvoEco-Paleo, FR-59000 Lille, France. E-mail: a.fisogni@gmail.com

Decision date: 03-Jul-2020

This article has been accepted for publication and undergone full peer review but has not been through the copyediting, typesetting, pagination and proofreading process, which may lead to differences between this version and the Version of Record. Please cite this article as doi: [10.1111/oik.07274]. 


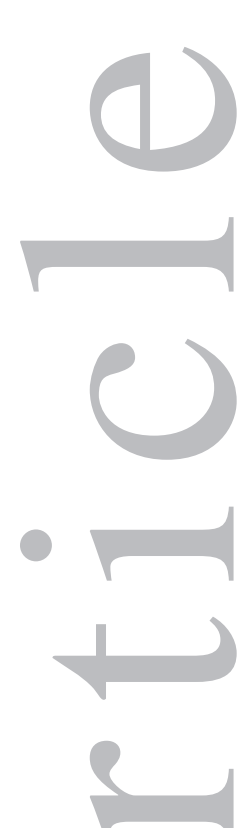

\section{Abstract}

Urbanization is one of the major threats to wild plants and pollinators, and its global increase demands a better understanding of the mechanism driving its negative impact. Urban warming and altered local environmental conditions have the potential to affect the timing of flowering and of pollinator activity. While previous evidence has shown that plant phenology tends to advance in urban areas, little is known about its effects on pollinator phenology. In this study we simultaneously assessed the response of the timing of flowering of native plants and of the flight period of wild pollinators to increased urbanization. We collected data from 12 sites along an urbanization gradient in Northern France, a region under strong anthropogenic pressure. Overall, we recorded more than 70 plant species, and we sampled more than 4300 wild bees and hoverflies belonging to 154 species. Plant flowering showed a strong response to urbanization at the community level with a striking advancement of the flowering peak in sites at high urbanization. On the contrary, pollinator communities did not show any clear shift of their flight phenology along the gradient, neither regarding abundance nor diversity. Our results indicate that phenologies of plant and pollinator communities can respond differently along the same urbanization gradient. These asymmetric responses can drive modifications in the structure of plant-pollinator networks, and potentially negatively affect the fitness of both mutualistic partners.

Keywords: flowering period; global change; hoverflies; land use; phenology; wild bees 


\section{Introduction}

The global proportion of urban areas and their human population have grown dramatically during the last century and are projected to grow further over the next decades (United Nations, Department of Economic and Social Affairs 2015). As a result of intensified human pressure, native animal and plant species are increasingly threatened by a variety of anthropogenic factors such as habitat modification and fragmentation, or the introduction of alien species (Williams et al. 2009, Adams and Lindsey 2011, Aronson et al. 2014). In addition, urban biodiversity must cope with altered local climates through increased temperatures ("urban heat island") and modified water regimes (Parlow 2011, McGrane 2016). Hence, urban species could experience greater negative effects of regional climate change compared to non-urban species (Ziska et al. 2003, Emilsson and Ode Sang 2017). The dynamics of urban biodiversity thus deserve more attention as they might help us to better assess the potential impacts of human activities on ecosystems.

Plant and pollinator communities can be significantly affected by urbanization. The replacement of soils with anthropogenic surfaces, together with the deliberate alteration of natural landscapes, act as filters that shape plant and pollinator community diversity and composition (McKinney 2008, Williams et al. 2009, Dunn and Heneghan 2011, Hamblin et al. 2017, 2018, Martins et al. 2017, Harrison et al. 2018). In particular, the urban heat island effect is regarded as a major driver of plant developmental events (i.e. their phenology, Roetzer et al. 2000, Neil and Wu 2006, Parlow 2011). Increased temperatures can drive shifts in plant phenologies by modifying the onset, peak or length of flowering, especially in annual insect-pollinated plant species that flower in spring (Fitter and Fitter 2002, Calinger et al. 2013, CaraDonna et al. 2014). In parallel, an augmentation of temperatures can significantly alter the phenology of insect species, 
mainly through physiological effects on winter diapause (Forister and Shapiro 2003, Altermatt 2010, Sgolastra et al. 2010). Analyses on long-term data suggest that plants and pollinators generally shift their phenologies at the same pace in response to increased global temperatures (Bartomeus et al. 2011, Rafferty and Ives 2011, Burkle et al. 2013, Ovaskainen et al. 2013). Nevertheless, phenological mismatches may occur and are expected to increase in the future in a context of global change (Gordo and Sanz 2005, Visser and Both 2005, Kudo and Ida 2013). Also, year-to-year variations in the flowering period of plants and timing of pollinator activity can induce single-year phenological mismatches, which can lead to a significant reduction of plant reproductive success (Kudo and Cooper 2019). While phenological shifts in urban habitats compared to their surroundings have been well documented for plants (Neil and $\mathrm{Wu}$ 2006), little is known regarding pollinator phenology in this environment (but see Leong et al. 2016, Harrison et al. 2018).

Uneven phenological shifts in plants and pollinators can lead to interaction mismatches (Ogilvie and Forrest 2017). If pollinators cannot cope with the changes of phenology of the plants they rely on, they could face shortage of resources with consequences on different fitness parameters (Schenk et al. 2018); at the same time, plants that lose their pollinators (e.g. because of phenological mismatch) may experience shortage of compatible pollen and reduced fruit or seed production (Thomson 2010, Rafferty and Ives 2012, 2013). Moreover, increased temperatures may cause physiological constraints on pollinators' activity (Scaven and Rafferty 2013, Hamblin et al. 2017), potentially modifying their foraging patterns and reducing their flight distance with negative consequences on pollen flow at larger scale. 
To date, the few studies that have analysed the joint responses of plants and pollinators have focused on the consequences of global climate change, while no study has yet considered parallel phenological changes in urban environments (Forrest 2015).

Moreover, recent works on pollinator phenology in urban environments have compared extremely contrasted landscapes, such as natural, agricultural and urban areas (Leong et al. 2016, Harrison et al. 2018), but not urban areas among them. In the present study, we compare plant and pollinator phenologies along a gradient of increasing urbanization in a context of strong anthropogenic pressure in Northern France, a region that has undergone intense human-mediated transformations over the last century (Toussaint et al. 2008, Deguines et al. 2014, Hautekèete et al. 2015). We simultaneously investigated the temporal dynamics of flowering abundance and diversity of native herbaceous plants, and the flight activity period of wild bee and hoverfly pollinator communities. By analysing these two components of mutualism simultaneously, we aim to evaluate whether they respond similarly to urbanization or whether there is potential for phenological uncoupling. 


\section{Materials and methods}

\section{Study sites and urbanisation gradient}

We performed this study in the Métropole Européenne de Lille, a densely populated territory $\left(1,139,929\right.$ inhabitants in $2014,1,749$ inhabitants $/ \mathrm{km}^{2}$; Insee, 2017) located in Northern France (Fig. 1). To compare study sites that vary in urbanization levels but with similar flowering plant communities, we focused on wildflower meadows that are homogeneously managed by the local Parks and Gardens Services throughout the Métropole area. These meadows were sown between 2010 and 2015, independently from this study, using a standardised seed mix containing only herbaceous plants native to the region (list of species included in the seed mix in Supplementary material Appendix 1 Table A1a, seeds purchased from Ecosem, Corroy-le-Grand, Belgium). Wildflower meadows were completely or partially mown at the beginning of summer (end of June - beginning of July) to avoid an overgrowth of Poaceae species and to favour a second flowering of some species in the late summer - early autumn. We selected 12 sites across an urbanization gradient based on the proportion of impervious surfaces within a $500 \mathrm{~m}$ buffer around the site edges (Fig. 1). Sites were not regular in shape, and sites 8 and 10 consisted of two meadows 5 and $10 \mathrm{~m}$ away from each other, respectively. Impervious surfaces (i.e. roads, buildings, parking lots, etc.) are a common proxy for urban development, and are related to an increase in temperature (Stewart and Oke 2012). A $500 \mathrm{~m}$ buffer covers the estimated maximum foraging distance for most wild bee species (Gathmann and Tscharntke 2002, Greenleaf et al. 2007, Zurbuchen et al. 2010, Wright et al. 2015) and hoverfly species (Lovei et al. 1998, Wratten et al. 2003). We used the geographical information system program ArcGIS 10.4 (ESRI 2011) to define the land cover types surrounding the study sites and to construct the gradient. We used the pre-existing land-use map "SCOT - Territorial coherence scheme" for the Métropole Européenne de Lille from 2015 (Agence de développement 
et d'urbanisme de Lille Métropole 2016, hereafter ADULM) as the baseline, and obtained a functional land cover at $5 \mathrm{~m}$ resolution by photo-interpreting aerial photographs taken in 2015 (www.ppige-npdc.fr). We considered sites with less than $50 \%$ cover of impervious surfaces as low urbanization sites (sites 1-4, mean impervious surface $\pm \mathrm{SD}=25.9 \pm 14.1$, Fig. 1 ), and those with more than $50 \%$ cover of impervious surfaces as the urban core (McKinney 2008). Sites in the urban core were further divided into medium (50-69\% impervious surfaces, sites 5-8, mean impervious areas $=$ $62.3 \pm 5.8)$ and high (70-100\% impervious surfaces, sites 9-12, mean impervious areas $=78.0 \pm 3.1$, Fig. 1) urbanization sites (Glaum et al. 2017). Therefore, although there were no similarly managed green areas in the highly urbanised city centre, the 12 selected sites displayed a range of cover by impervious surface from 8.6 to $80.5 \%$. More details about site characteristics are reported in Supplementary material Appendix 1 Table A2.

\section{Flowering abundance and diversity}

We visited each of the 12 sites every other week from April $4^{\text {th }}$ to June $29^{\text {th }} 2017$. We visited three sites per day, with all sites being visited within 4-10 days (median: 4 days), depending on weather conditions. At each sampling event, we identified all plant species in flower with the help of a dichotomous key (Lambinon et al. 2004). None of the few trees present in some of the study sites flowered during our samplings, and were therefore excluded from the analysis. No shrubs were present in any of the study sites. We included in the analysis all herbaceous flowering plants encountered, including species growing from both the seed bank and from the seed mix sown by local practitioners. We chose a simplified method that combines information on flower cover and flower quantity. First, we estimated the total proportion of surface covered by floral units (sensu Gibson et al. 2006) of all flowering species at each study site. Then we 
estimated the relative abundance of each flowering species. To rank flowering species we used Braun-Blanquet's coefficients of abundance-dominance, ranging from $i$ to 5 for least to most abundant respectively (van der Maarel 1979, Mucina et al. 2000). We then converted these coefficients in percentage intervals and in mean values of percentage cover classes: $i$ - 1 individual, +- few individuals less than $1 \%, 1-1-10 \%, 2-11-25 \%$, $3-26-50 \%, 4-51-75 \%, 5-76-100 \%$ (de Manincor et al. 2020). All estimations were performed by the same observer. We defined flowering period as the period included between the first and the last day a plant has been observed in flower, and flowering peak as the highest percentage of flower cover estimated throughout the season. We defined the number of plant species in flower at each survey as plant flowering richness.

\section{Pollinator sampling}

Pollinators were sampled on the same days we recorded flower abundances, with similar and favourable weather conditions in all sites at each sampling event (clear sky or scattered clouds, maximum temperatures $>15^{\circ} \mathrm{C}$, and low wind). We focused on the two major pollinator groups at middle European latitudes (Ollerton 2017): bees (Hymenoptera: Anthophila) and hoverflies (Diptera: Syrphidae). Even though we did not measure pollination efficiency, these taxa have the potential to be pollinators to some extent of at least some of the visited plants in the study sites, therefore we refer to them as pollinators throughout the text for simplicity. We used pan trap and hand net captures at the same time to increase sampling efficiency for both bees and hoverflies (Leong and Thorp 1999, Laubertie et al. 2006, Roulston et al. 2007, Grundel et al. 2011, Joshi et al. 2015). Pan traps consisted of plastic bowls painted with UV-bright white, blue and yellow paint (Sparvar Leuchtfarbe, spray-color GmbH, Germany), following the widely used method recommended by Westphal et al. (2008). At each sampling date, three clusters including one trap per colour (nine traps in total) were placed in all 
the studied sites, and were activated in the morning (between 9 a.m. and 11:30 a.m.)

with soapy water $(200 \mathrm{~mL})$ to avoid the escape of insects. Pan traps were set out for at least $6 \mathrm{~h}$ (median: $7 \mathrm{~h}$ ). Insects captured with pan traps were collected from all sites in the late afternoon of the same day (between 3:30 p.m. and 6:30 p.m.), and stored in ethanol $70 \%$ until pinning. In addition to pan traps, we set up variable transects in which two operators randomly walked each site for 45 minutes and collected the pollinators encountered, independently of where they were found (i.e. on the bare ground, on the vegetation, or visiting a flower). Transects started around 11 a.m. at the first site visited in the morning, around 1 p.m. at the second site and around 3 p.m. at the third site. Insects sampled with hand nets were put in killing vials with ethyl acetate, and transferred to the laboratory for pinning. We changed the order of site visits within each field session to reduce the effect of daily weather differences on insect activity (details on visiting sequences in Supplementary material Appendix 1 Table A2). We did not detect any persistent trend in the measured temperature or relative humidity (measured during visits using a Tinytag Plus 2 data logger), among site triplets visited at each sampling event, nor between classes of urbanization (Supplementary material Appendix 2 Fig. A1). All sampled specimens were later identified to the species level by expert taxonomists (list of specialists is reported in the Acknowledgement section) and deposited at the [anonymized for double-blind peer review]. We excluded Apis mellifera since its presence, and therefore apparent phenology, is strongly related to human activity (i.e. beekeeping).

\section{Data analysis}

All statistical analyses were performed in R version 3.4.3 (R Core Team, 2019). To analyse the overall homogeneity of flowering plant species composition among sites, we used latent block models (LBMs) based on presence - absence data and Bernoulli 
distribution (R package blockmodels; INRA and Leger, 2015). LBMs are probabilitybased models that simultaneously cluster sites (rows) and species (columns) based on latent blocks (Govaert and Nadif 2008, Keribin et al. 2015, Thomas et al. 2015). An incidence matrix was generated to highlight homogeneous blocks of sites and species (Supplementary material Appendix 3 Fig. A1a). We performed the same analysis on the presence - absence of pollinators in the 12 sites (Supplementary material Appendix 3 Fig. A1b).

To analyse temporal changes in the flowering species community composition among the three urbanization classes, we performed LBMs with quantitative data and Poisson distribution (INRA and Leger 2015).To carry out this analysis we used six categorical variables for the sampling dates (i.e. early and late April, May and June, respectively). First, we performed LBMs on the proportional flower cover of each species (columns) found at each sampling date in each site (rows, Supplementary material Appendix 3 Fig. A2a). Second, we calculated the average date $x_{j}$ at which plant $j$ was flowering as:

$$
x_{j}=\frac{\sum_{i} a_{i j} t_{i}}{a_{i j}}
$$

where $t_{i}$ is the date of sampling in site $i$ and $a_{i j}$ is the flower cover of plant $j$ in site $i$ and time $t_{i}$. Third, we calculated the average date of flowering of each 'site $\times$ sampling date' block $K$ retrieved by the LBMs (Supplementary material Appendix 3 Fig. A2a) as:

$$
y_{K}=\frac{\sum_{j} \sum_{i \in I(K)} a_{i j} x_{j}}{\sum_{j} \sum_{i \in I(K)} a_{i j}}
$$

where $I(K)$ represents the set of sites $\times$ sampling dates corresponding to block $K$. Finally, we ordered blocks according to flowering sequence and we created one heat map (packages 'ggplot2' and 'viridis', Wickham 2016; Garnier 2018) for each urban 
class with tiles coloured as a function of site density (i.e. the number of sites included at any time $t_{i}$ in a given block). We performed the same analysis for pollinators using the abundance of each insect species found in each site at each sampling date and calculating the average date of insect flying activity for each 'site $\times$ sampling date' block as retrieved by LBMs (Supplementary material Appendix 3 Fig. A2b).

We modelled the temporal variation of several response variables in the three urbanization classes by means of generalized linear mixed models (GLMMs; function glmmadmb, Skaug et al. 2018) using the general formula:

Variable $\sim$ urban + day $+d a y^{2}+$ urban: day + urban: $d a y^{2}+(1 \mid$ site $)$

where urban is the urbanization class (low, medium or high), and day is the Julian day of the year $\left(1^{\text {st }}\right.$ of January $=1,31^{\text {st }}$ of December $\left.=365\right)$. We included $d a y^{2}$ to allow for non-linear effects, and site as random effect to account for the variability among sites within a same urban class. We chose the best model(s) based on goodness-of-fit using the Akaike Information Criterion with correction for small sample sizes (AICc), and by selecting the model(s) with the lowest AICc value (Burnham and Anderson 2002). If $\triangle \mathrm{AICc}$ values between some models were lower than 4 , we used model averaging to calculate a weighted average of the parameter estimates ( $\mathrm{R}$ package MuMIn, Bartoń 2018), based on Akaike weights proportional to $\exp (-\Delta \mathrm{AICc} / 2)(\mathrm{Burnham}$ and Anderson 2002, Massol et al. 2007). Finally, we calculated 95\% jackknife confidence intervals for the estimated model fits.

We modelled total community flowering cover and cover of the ten most common flowering species using GLMMs with Gaussian error distribution (Supplementary material Appendix 3 Fig. A3) on relative abundances $p$ (abundances divided by their maximum site-wise value throughout the season), further transformed into "almost logit" variables $y$ to approximate a Gaussian distribution following:

$y=\log \left(\frac{p+0.001}{1.001-p}\right)$ 
We modelled total pollinator abundance and the abundance of the six pollinator families found in this study using GLMMs with negative binomial distribution and logarithm link (Supplementary material Appendix 3 Fig. A4), using the number of sampled pollinators at each sampling date as response variable. We modelled flowering and pollinator richness using GLMMs with binomial error distribution (logit-link) using the number of species found at each sampling event as the response variable. For models of species richness, we excluded rare flowering species (i.e. species included in block 3 , Supplementary material Appendix 3 Fig. A2a) and pollinator species found as singletons (single occurrence) because the temporal distribution of these species was not informative on potential phenological mismatches. Using binomial error, rather than Poisson, allowed us to obtain more conservative estimates of standard error for mean species richness (i.e. without assuming that the mean and variance of species richness coincide). 


\section{Results}

We conducted a total of 69 field sessions at the 12 study sites along the three-month study period (87 days). All sites were visited six times, with the exception of sites 5, 6 and 10 (Fig. 1), which were visited five times because of prolonged bad weather from mid to late April.

\section{Plant and pollinator communities}

Overall, we found a total of 74 flowering plant species, all native to the region, including both species originating from the seed mix and species developed from the local seed bank (Supplementary material Appendix 1 Table A1a). We sampled a total of 4,317 pollinator individuals (3,601 bees, 716 hoverflies) belonging to 6 families, 43 genera (19 bee and 24 hoverfly genera) and 154 species (102 bee and 52 hoverfly species). Overall, $78.6 \%$ of the observed species interacted with species from the other trophic level (80\% of plants, $77.9 \%$ of pollinators, [cross-reference anonymized for double-blind peer review]). The list of pollinator species is reported in Supplementary material Appendix 1 Table A1b, and their abundance at each site $\times$ sampling event is available in the Digital Repository.

The overall flowering plant community composition was homogeneous among sites (i.e. only one block was retrieved for sites in the LBM, Supplementary material Appendix 3 Fig. A1a), while plant species differed in their occurrence patterns -independently from urbanization classes- and could be classified into three groups (three blocks for plants in the LBM: one group containing the ten most common flowering species, a second group the less common species and a third group the rare species, Supplementary material Appendix 3 Fig. A1a). Similarly, the overall pollinator community composition was homogeneous among sites, while two blocks were found for common and less 
common pollinator species, respectively (Supplementary material Appendix 3 Fig. A1b).

\section{Phenology of flowering plants and pollinators}

LBMs on quantitative data retrieved 15 'site $\times$ sampling date' blocks for the plant community and 7 for the pollinator community (Fig. 2, Supplementary material Appendix 3 Fig. A2a-b). Heat maps highlighted an effect of urbanization on the flowering plant community composition: similar plant communities (i.e. belonging to a given block) tended to flower earlier at medium and high urbanization compared to low urbanization (Fig. 2a). On the contrary, no clear phenological shifts appeared for the pollinator community among urbanization classes (Fig. 2b).

Urbanization had a strong effect on flowering phenology at the whole plant community level (significant effect of the interactions 'urban: day' and 'urban: day' ${ }^{2}$ ' in the best model, Table 1, complete model coefficients in Supplementary material Appendix 4 Table A1). The estimated flowering peak occurred about four weeks earlier in high urbanization sites than in low urbanization sites (Fig. 3a). Flowering began earlier and the proportion of flower cover increased faster in medium than in low urbanization sites, although the flowering peaks occurred at about the same time (Fig. 3a). Similar patterns were found for the majority of the ten most common flowering species, with a general advancement of flowering in highly urbanised sites, while four species did not show any phenological changes (Supplementary material Appendix 3 Fig. A3,

Supplementary material Appendix 4 Table A1, A2). The peak of flowering richness occurred considerably earlier at both medium and high urbanization than at low urbanization (Fig. 3c).

Whole pollinator community abundance increased throughout the season and did not show significant temporal shifts among urbanization classes (Fig. 3b, Table 1). Similar 
patterns were observed for pollinator families, independently of their phenology (i.e. early or late pollinators), with only rare weak interaction effects between date and urbanization class (Supplementary material Appendix 3 Fig. A4, Supplementary material Appendix 4 Table A1, A2). Total pollinator richness increased moderately over time and did not differ between urbanization classes, although it was always slightly lower at high urbanization than at medium or low urbanization (Fig. 3d). 


\section{Discussion}

We found that increased urbanization strongly affected the timing of flowering but not of pollinators' flying activity. Plant species communities showed a striking advancement of the peak of flowering in high urbanization sites compared to low urbanization sites, as well as an earlier peak of floral resource diversity. On the contrary, pollinator communities did not display clear patterns of temporal shifts in their abundance or diversity.

Plant flowering phenology may shift in time via plasticity or directional selection as an adaptation to changes in light and temperature conditions (Anderson et al. 2012, Richardson et al. 2017), which are typically intensified in urban environments (Stewart and Oke 2012, Hale et al. 2013). The early onset of flowering that we have observed in highly urbanised sites compared to low urbanised sites agrees with other studies that showed similar responses of flowering to an increased urbanization (Roetzer et al. 2000, Neil and Wu 2006). Moreover, we detected not only a faster increase of the proportion of flower cover along the gradient, but also a clear shift in the timing of flowering peak among the three urban classes. This advancement of the spring phases along an urban gradient - that is, without considering highly contrasted areas (e.g. natural vs. agricultural vs. urban) - emphasizes the major impact of urbanization on plant flowering phenology.

Contrarily to flowering plants, we did not observe apparent changes in the phenology of pollinator communities along the gradient of urbanization with respect to either their abundance or richness. The perceived asymmetric impacts of urbanization on plants and pollinators can be partially related to a differential phenotypic plasticity, e.g. a stronger phenological response to changes in spring temperatures in plants than in pollinators 
(Iler et al. 2013, Kudo and Ida 2013, Kudo 2014, Pyke et al. 2016). This can also reflect responses to different cues (e.g. temperature $v s$ photoperiod), or to similar stimuli that display fine-scale geographical variation. Plants, being sessile, may then respond to the local (micro)climate in the timing of flowering. Conversely, bees and hoverflies might respond to microclimatic cues of possibly distant nesting sites as well as to the proximity and availability of floral resources outside the study areas. The mobility of pollinators can allow them to track flower resources over time within a certain spatial range, which can confound the effects of local conditions at the study sites, especially for species with a wider range of flight. However, the mobility of pollinators with potential long flights (e.g. bumblebees) can be limited in urban environments (Van Rossum and Triest 2010), suggesting that land use at the local scale plays a major role in determining the response of pollinator phenology.

Whatever the causes, our results show a potential mismatch between plant flowering phenology and pollinator activity along the studied gradient of urbanization.

Phenological mismatches can disrupt plant-pollinator networks (Memmott et al. 2007), and can potentially negatively affect the fitness of both mutualistic partners if they face a shortage of pollen vectors or foraging resources (Schenk et al. 2018, Kudo and Cooper 2019). The mismatch observed in this study has likely driven modifications in the structure of plant-pollinator interaction networks at different levels of urbanization ([cross-reference anonymized for double-blind peer review]), and can be expected to result in reduced pollination services in urban areas (Rivkin et al. 2020). However, the relatively constant abundance and high diversity of the pollinator community recorded in the study sites throughout the season - more than 150 species sampled in a threemonth study period - could favour functional redundancy and may play an important 
role in ensuring an overall phenological synchrony and an adequate pollen supply to flowering plants (Bartomeus et al. 2013, Theodorou et al. 2017).

The relatively high abundance of flowering plants observed at the study sites might support the local populations of pollinators along the urban gradient. We have found both specialist and generalist pollinators visiting flowers from spring to early summer ([cross-reference anonymized for double-blind peer review]). It is important to note that the management of the sites foresees the use of native plants, ensuring the presence of species with abundant floral resources at different time periods (e.g. Taraxacum sp., Leucanthemum vulgare and Centaurea jacea; Hicks et al. 2016), but also requires a summer mowing to reduce the emergence of weeds and encourage a second flowering in early autumn. This type of management can produce a gap in foraging resource availability during summer, and can interact with the early decline in flowering observed in highly urban areas. However, pollinators can feed on other species outside the study sites, for example on trees, ornamental plants, or in public and private gardens (Frankie et al. 2005, Banaszak-cibicka and Żmihorski 2012, Somme et al. 2016), and the advancement of phenology in urban areas may not be fatal especially for species active late in the season.

In this study we could not separate the effects of abiotic (e.g. increased temperatures, soil humidity, rain intensity, air and light pollution) and biotic factors (e.g. biotic interactions, genetic variation), which likely interact in determining the phenology of the studied organisms along the gradient of urbanization (Leong et al. 2016, Wohlfahrt et al. 2019). Future studies directly addressing multiple factors might help determine which are more important in shaping the observed patterns. Moreover, since the heat island intensity correlates positively with the size of the city (Parlow 2011), studies that 
include urban areas of different sizes could highlight different responses of plant and pollinator phenologies. Another limitation of this study concerns our inability to evaluate pairwise differences in phenology that would drive more specific disruptions of function (e.g. pollination). Species-level responses can differ from community-level responses (Iler et al. 2013), and specialist species that closely depend on each other are likely more prone to a disruption of interactions than generalist species if their partners are not available at a given time.

Here, we show that the phenology of plant and pollinator communities responds differently to a gradient from less to more urbanized areas. The flowering peak and diversity were significantly advanced in sites at high urbanization, while pollinators did not display shifts overall. These asymmetric responses can modify the patterns of plantpollinator interactions, and potentially drive a disruption of the pollination function. However, the relatively constant temporal abundance and diversity of pollinators coupled with a high availability of floral resources provided by local practitioners should offer reassurance. Our results can also inform decision-makers about the type of management of urban green areas and their value to local pollinators. Future studies should investigate the link between changes in phenology and function in the urban environment, and focus on specific interactions to highlight which species are more susceptible to mismatch.

\section{Data and code availability statement}

All code and data are available from the Zenodo Digital Repository: < http://doi.org/10.5281/zenodo.3606004 > (Fisogni et al. 2020)

\section{Acknowledgements}


We thank Yohan Tison and Olivier Adalberon for helping with the choice of study sites in the city of Lille, and Samuel Nef for the support with sites in the Métropole Européenne de Lille territory. We thank Amélie Peaudecerf, Roman Stetsenko and Alexis Parret for valuable help with field work and insect preparation. We are grateful to Jens d'Haeseleer for the identification of bees of the family Andrenidae, Megachilidae and Colletidae, to Alain Pauly for the identification of Halictidae, and to Morgane Follschweiller and Pierre Rasmont for validating bumblebee identifications made by AF. Syrphidae were identified by CV. We are grateful to the four anonymous reviewers that provided constructive comments on previous drafts of the manuscript.

\section{Funding}

The authors thank the Région Hauts-de-France, and the Ministère de l'Enseignement Supérieur et de la Recherche (CPER Climibio), and the European Fund for Regional Economic Development for their financial support. The authors also thank the ANR ARSENIC project (grant no. 14-CE02-0012) and EOS Clips Project (grant n³0947854) for financial support.

\section{Conflict of interest}

The authors declare no conflict of interest.

Title: Urbanization drives an early spring for plants but not for pollinators 


\section{References}

Adams, C. and Lindsey, K. 2011. Anthropogenic ecosystems: the influence of people on urban wildlife populations. - In: Niemelä, J. (ed), Urban Ecology. Patterns, processes, and applications. 1st ed.n. Oxford University Press, pp. 116-128.

Agence de développement et d'urbanisme de Lille Métropole (ADULM) 2016. SCOT de Lille Métropole. Occupation du sol en 2015.

Altermatt, F. 2010. Tell me what you eat and I'll tell you when you fly: diet can predict phenological changes in response to climate change. - Ecol. Lett. 13: 1475-1484.

Anderson, J. T. et al. 2012. Phenotypic plasticity and adaptive evolution contribute to advancing flowering phenology in response to climate change. - Proc. R. Soc. B Biol. Sci. 279: 3843-3852.

Aronson, M. F. J. et al. 2014. A global analysis of the impacts of urbanization on bird and plant diversity reveals key anthropogenic drivers. - Proc. R. Soc. B 281: 20133330.

Banaszak-cibicka, W. and Żmihorski, M. 2012. Wild bees along an urban gradient: winners and losers. - J. Insect Conserv. 16: 331-343.

Bartomeus, I. et al. 2011. Climate-associated phenological advances in bee pollinators and bee-pollinated plants. - Proc. Natl. Acad. Sci. 108: 20645-20649.

Bartomeus, I. et al. 2013. Biodiversity ensures plant-pollinator phenological synchrony against climate change. - Ecol. Lett. 16: 1331-1338.

Bartoń, K. 2019. MuMIn: Multi-Model Inference. R package version 1.43.15. https://CRAN.R-project.org/package=MuMIn.

Burkle, L. A. et al. 2013. Plant-pollinator interactions over 120 years: loss of species, co-occurrence, and function. - Science 340: 1611-1615.

Burnham, K. and Anderson, D. 2002. Information and Likelihood theory: A Basis for Model Selection and Inference. - In: Model selection and multimodel inference: a 
practical information-theoretic approach. 2nd ed.n. Springer-Verlag, pp. 49-97.

Calinger, K. M. et al. 2013. Herbarium specimens reveal the footprint of climate change on flowering trends across north-central North America. - Ecol. Lett. 16: 10371044.

CaraDonna, P. J. et al. 2014. Shifts in flowering phenology reshape a subalpine plant community. - Proc. Natl. Acad. Sci. 111: 4916-4921.

de Manincor, N. et al. 2020. Does phenology explain plant-pollinator interactions at different latitudes? An assessment of its explanatory power in plant-hoverfly networks in French calcareous grasslands. - Oikos 129: 753-765.

Deguines, N. et al. 2014. Large-scale trade-off between agricultural intensification and crop pollination services. - Front. Ecol. Environ. 12: 212-217.

Dunn, C. and Heneghan, L. 2011. Composition and diversity of urban vegetation. - In: Niemelä, J. (ed), Urban Ecology. Patterns, processes, and applications. 1st ed.n. Oxford University Press, pp. 103-115.

Emilsson, T. and Ode Sang, Å. 2017. Impacts of climate change on urban areas and nature-based solutions for adaptation. - In: Kabisch, N. et al. (eds), Nature-Based Solutions to Climate Change Adaptation in Urban Areas: Linkages between Science, Policy and Practice. Springer International Publishing, pp. 15-27.

ESRI 2011. ArcGIS Desktop: Release 10.4. Redlands, CA: Environmental Systems Research Institute.

Fitter, A. and Fitter, R. 2002. Rapid changes in flowering times in British plants. Science 296: 1689-1691.

Forister, M. and Shapiro, A. 2003. Climatic trends and advancing spring flight of butterflies in lowland California. - Glob. Chang. Biol. 9: 1130-1135.

Forrest, J. R. 2015. Plant - pollinator interactions and phenological change : what can we learn about climate impacts from experiments and observations? - Oikos 124: 
$4-13$.

Frankie, G. W. et al. 2005. Ecological patterns of bees and their host ornamental flowers in two northern California cities. - J. Kansas Entomol. Soc. 78: 227-246.

Garnier, S. 2018. viridis: default color maps from 'matplotlib'. R package version 0.5.1. https://CRAN.R-project.org/package=viridis.

Gathmann, A. and Tscharntke, T. 2002. Foraging ranges of solitary bees. - J. Anim. Ecol. 71: 757-764.

Gibson, R. H. et al. 2006. Pollinator webs, plant communities and the conservation of rare plants: arable weeds as a case study. - J. Appl. Ecol. 43: 246-257.

Glaum, P. et al. 2017. Big city Bombus: using natural history and land-use history to find significant environmental drivers in bumble-bee declines in urban development. - R. Soc. Open Sci. 4: 170156.

Gordo, O. and Sanz, J. 2005. Phenology and climate change: a long-term study in a Mediterranean locality. - Oecologia 146: 484-495.

Govaert, G. and Nadif, M. 2008. Block clustering with Bernoulli mixture models: comparison of different approaches. - Comput. Stat. Data Anal. 52: 3233-3245.

Greenleaf, S. S. et al. 2007. Bee foraging ranges and their relationship to body size. Oecologia 153: 589-596.

Grundel, R. et al. 2011. Effectiveness of bowl trapping and netting for inventory of a bee community. - Environ. Entomol. 40: 374-380.

Hale, J. D. et al. 2013. Mapping lightscapes: spatial patterning of artificial lighting in an urban landscape. - PLoS One 8: e61460.

Hamblin, A. L. et al. 2017. Physiological thermal limits predict differential responses of bees to urban heat-island effects. - Biol. Lett. 13: 20170125.

Hamblin, A. L. et al. 2018. Wild bee abundance declines with urban warming, regardless of floral density. - Urban Ecosyst. 21: 419-428. 
Harrison, T. et al. 2018. Forest bees are replaced in agricultural and urban landscapes by native species with different phenologies and life-history traits. - Glob. Chang. Biol. 24: 287-296.

Hautekèete, N.-C. et al. 2015. Habitat type shapes long-term plant biodiversity budgets in two densely populated regions in north-western Europe. - Divers. Distrib. 21: $631-642$.

Hicks, D. M. et al. 2016. Food for pollinators: quantifying the nectar and pollen resources of urban flower meadows. - PLoS One 11: e0158117.

Iler, A. M. et al. 2013. Maintenance of temporal synchrony between syrphid flies and floral resources despite differential phenological responses to climate. - Glob. Chang. Biol. 19: 2348-2359.

INRA and Leger, J.-B. 2015. blockmodels: Latent and Stochastic Block Model Estimation by a "V-EM" Algorithm. R package version 1.1.1. https://CRAN.Rproject.org/package=blockmodels.

Insee 2017. La population des territoires de la région Hauts-de-France au 1 er janvier 2014. - In: Huart, D. and Lhuillier, N. (eds), Insee Dossier Hauts-de-France, pp. 176.

Joshi, N. K. et al. 2015. Comparative trapping efficiency to characterize bee abundance, diversity, and community composition in apple orchards. - Ann. Entomol. Soc. Am. 108: 785-799.

Keribin, C. et al. 2015. Estimation and selection for the latent block model on categorical data. - Stat. Comput. 25: 1201-1216.

Kudo, G. 2014. Vulnerability of phenological synchrony between plants and pollinators in an alpine ecosystem. - Ecol. Res. 26: 571-581.

Kudo, G. and Ida, T. Y. 2013. Early onset of spring increases the phenological mismatch between plants and pollinators. - Ecology 94: 2311-2320. 
Kudo, G. and Cooper, E. J. 2019. When spring ephemerals fail to meet pollinators: mechanism of phenological mismatch and its impact on plant reproduction. - Proc. R. Soc. B 286: 20190573.

Lambinon, J. et al. 2004. Nouvelle flore de la Belgique, du Grand-Duché de Luxembourg, du Nord de la France et des Régions voisines. - Edition du Jardin botanique national de Belgique.

Laubertie, E. et al. 2006. The role of odour and visual cues in the pan-trap catching of hoverflies (Diptera: Syrphidae). - Ann. Appl. Biol. 148: 173-178.

Leong, J. and Thorp, R. 1999. Colour-coded sampling: the pan trap colour preferences of oligolectic and nonoligolectic bees associated with a vernal pool plant. - Ecol. Entomol. 24: 329-335.

Leong, M. et al. 2016. Temporal dynamics influenced by global change: bee community phenology in urban, agricultural, and natural landscapes. - Glob. Chang. Biol. 22: $1046-1053$.

Lovei, G. et al. 1998. Dispersal and effects of barriers on the movement of the New Zealand hover fly Melanostoma fasciatum (Dipt., Syrphidae) on cultivated land. J. Appl. Entomol. 122: 115-120.

Martins, K. T. et al. 2017. Patterns of pollinator turnover and increasing diversity associated with urban habitats. - Urban Ecosyst. 20: 1359-1371.

Massol, F. et al. 2007. The influence of trophic status and large-scale climatic change on the structure of fish communities in Perialpine lakes. - J. Anim. Ecol. 76: 538-551. McGrane, S. J. 2016. Impacts of urbanisation on hydrological and water quality dynamics , and urban water management: a review. - Hydrol. Sci. J. 61: 22952311.

McKinney, M. 2008. Effects of urbanization on species richness: a review of plants and animals. - Urban Ecosyst. 11: 161-176. 
Memmott, J. et al. 2007. Global warming and the disruption of plant-pollinator interactions. - Ecol. Lett. 10: 710-717.

Mucina, L. et al. 2000. Common data standards for recording relevés in field survey for vegetation classification. - J. Veg. Sci. 11: 769-772.

Neil, K. and Wu, J. 2006. Effects of urbanization on plant flowering phenology: a review. - Urban Ecosyst. 9: 243-257.

Ogilvie, J. E. and Forrest, J. R. 2017. Interactions between bee foraging and floral resource phenology shape bee populations and communities. - Curr. Opin. Insect Sci. 21: $75-82$.

Ollerton, J. 2017. Pollinator diversity: distribution, ecological function, and conservation. - Annu. Rev. Ecol. Evol. Syst. 48: 353-376.

Ovaskainen, O. et al. 2013. Community-level phenological response to climate change. - Proc. Natl. Acad. Sci. 110: 13434-13439.

Parlow, E. 2011. Urban climate. - In: Niemelä, J. (ed), Urban Ecology. Patterns, processes, and applications. 1st ed.n. Oxford University Press, pp. 31-44.

Pyke, G. H. et al. 2016. Effects of climate change on phenologies and distributions of bumble bees and the plants they visit. - Ecosphere 7: e01267.

R Core Team 2019. R: A language and environment for statistical computing. $R$ Foundation for Statistical Computing, Vienna, Austria. https://www.R-project.org/

Rafferty, N. E. and Ives, A. R. 2011. Effects of experimental shifts in flowering phenology on plant-pollinator interactions. - Ecol. Lett. 14: 69-74.

Rafferty, N. E. and Ives, A. R. 2012. Pollinator effectiveness varies with experimental shifts in flowering time. - Ecology 93: 803-814.

Rafferty, N. E. and Ives, A. R. 2013. Phylogenetic trait-based analyses of ecological networks. - Ecology 94: 2321-2333.

Richardson, B. A. et al. 2017. Will phenotypic plasticity affecting flowering phenology 
keep pace with climate change? - Glob. Chang. Biol. 23: 2499-2508.

Rivkin, L. R. et al. 2020. Variation in pollinator- mediated plant reproduction across an urbanization gradient. - Oecologia 192: 1073-1083.

Roetzer, T. et al. 2000. Phenology in central Europe - differences and trends of spring phenophases in urban and rural areas. - Int. J. Biometeorol. 44: 60-66.

Roulston, T. H. et al. 2007. A comparison of pan trap and intensive net sampling techniques for documenting a bee (Hymenoptera: Apiformes) fauna. - J. Kansas Entomol. Soc. 80: 179-181.

Scaven, V. L. and Rafferty, N. E. 2013. Physiological effects of climate warming on flowering plants and insect pollinators and potential consequences for their interactions. - Curr. Zool. 59: 418-426.

Schenk, M. et al. 2018. Desynchronizations in bee - plant interactions cause severe fitness losses in solitary bees. - J. Anim. Ecol. 87: 139-149.

Sgolastra, F. et al. 2010. Effect of temperature regime on diapause intensity in an adultwintering Hymenopteran with obligate diapause. - J. Insect Physiol. 56: 185-194.

Skaug, A. H. et al. 2018. Generalized Linear Mixed Models using 'AD Model Builder'. R package version 0.8.3.3. http://glmmadmb.r-forge.

Somme, L. et al. 2016. Food in a row: urban trees offer valuable floral resources to pollinating insects. - Urban Ecosyst. 19: 1149-1161.

Stewart, I. and Oke, T. 2012. Local climate zones for urban temperature studies. - Bull. Am. Meteorol. Soc. 93: 1879-1900.

Theodorou, P. et al. 2017. The structure of flower visitor networks in relation to pollination across an agricultural to urban gradient. - Funct. Ecol. 31: 838-847.

Thomas, M. et al. 2015. A network-based method to detect patterns of local crop biodiversity: validation at the species and infra-species levels. - Adv. Ecol. Res. 53: 259-320. 
Thomson, J. D. 2010. Flowering phenology, fruiting success and progressive deterioration of pollination in an early-flowering geophyte. - Philos. Trans. R. Soc. B 365: 3187-3199.

Toussaint, B. et al. 2008. Flore de la Flandre française. Centre régional de phytosociologie agréé Conservatoire botanique national de Bailleul.

United Nations, Department of Economic and Social Affairs, P. D. 2015. World urbanization prospects: the 2014 revision, (ST/ESA/SER.A/366).

van der Maarel, E. 1979. Transformation of cover-abundance values in phytosociology and its effects on community similarity. - Vegetatio 39: 97-114.

Van Rossum, F. and Triest, L. 2010. Pollen dispersal in an insect-pollinated wet meadow herb along an urban river. - Landsc. Urban Plan. 95: 201-208.

Visser, M. E. and Both, C. 2005. Shifts in phenology due to global climate change: The need for a yardstick. - Proc. R. Soc. B 272: 2561-2569.

Westphal, C. et al. 2008. Measuring bee diversity in different European habitats and biogeographical regions. - Ecol. Monogr. 78: 653-671.

Wickham, H. 2016. ggplot2: elegant graphics for data analysis. Springer-Verlag New York.

Williams, N. S. G. et al. 2009. A conceptual framework for predicting the effects of urban environments on floras. - J. Ecol. 97: 4-9.

Wohlfahrt, G. et al. 2019. The urban imprint on plant phenology. - Nat. Ecol. Evol. 3: $1668-1674$.

Wratten, S. et al. 2003. Field boundaries as barriers to movement of hover flies (Diptera: Syrphidae) in cultivated land. - Oecologia 134: 605-611.

Wright, I. R. et al. 2015. Evidence of forage distance limitations for small bees (Hymenoptera: Apidae). - Eur. J. Entomol. 112: 303-310.

Ziska, L. H. et al. 2003. Cities as harbingers of climate change: Common ragweed, 
urbanization, and public health. - J. Allergy Clin. Immunol. 111: 290-295.

Zurbuchen, A. et al. 2010. Long foraging distances impose high costs on offspring production in solitary bees. - J. Anim. Ecol. 79: 674-681. 


\section{Figure Legends}

Figure 1. Map of the twelve study sites in the Métropole Européenne de Lille, France.

Urbanization classes are based on the percentage cover of impervious surfaces within a 500 m buffer: low urbanization (blue dots) $<50 \%$, medium urbanization (yellow dots) $50 \%<x<70 \%$, high urbanization (orange dots) $\geq 70 \%$. Light and dark green represent agricultural and green areas, respectively. Light and dark grey represent impervious surfaces. Blue represents water surfaces. Site characteristics are reported in Supplementary material Appendix 1 Table A2.

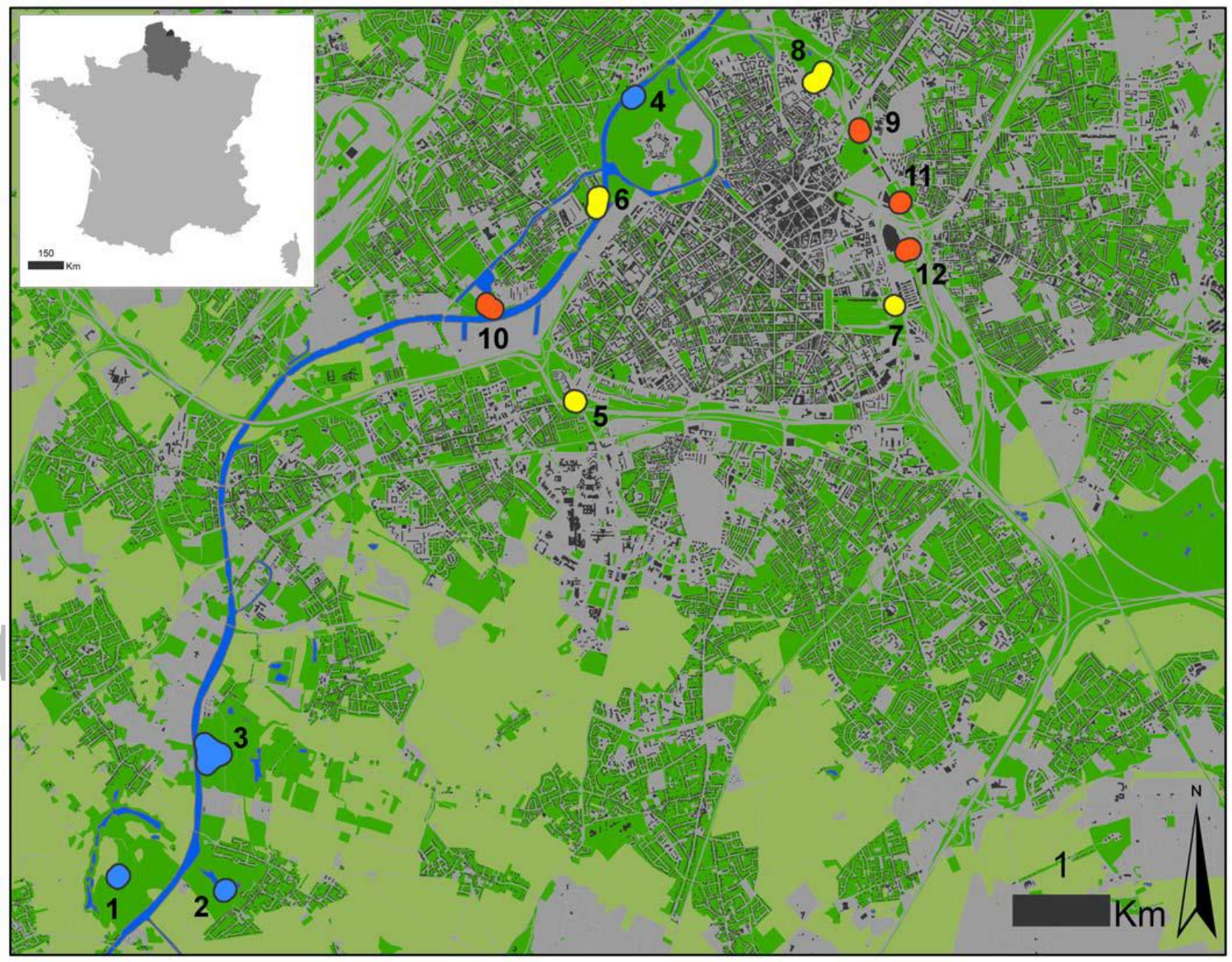

'This article is protected by copyright. All rights reserved.' 
Figure 2. Heat map of the (a) flowering plant and (b) pollinator community phenology in the three urbanization classes obtained from the LBMs (latent block models). Each tile represents a 'site $\times$ sampling date' block, as retrieved from LBMs (letters correspond to blocks in Supplementary material Appendix 3 Fig. A2a-b), at a given sampling date (1-2: early and late April, 3-4: early and late May, 5-6: early and late June). Tile colours are a function of site density (i.e. the number of sites occurring within a given block). Sites with similar community composition showed advanced phenology at higher urbanization for plants (diagonal shifted to the left), but not for pollinators.

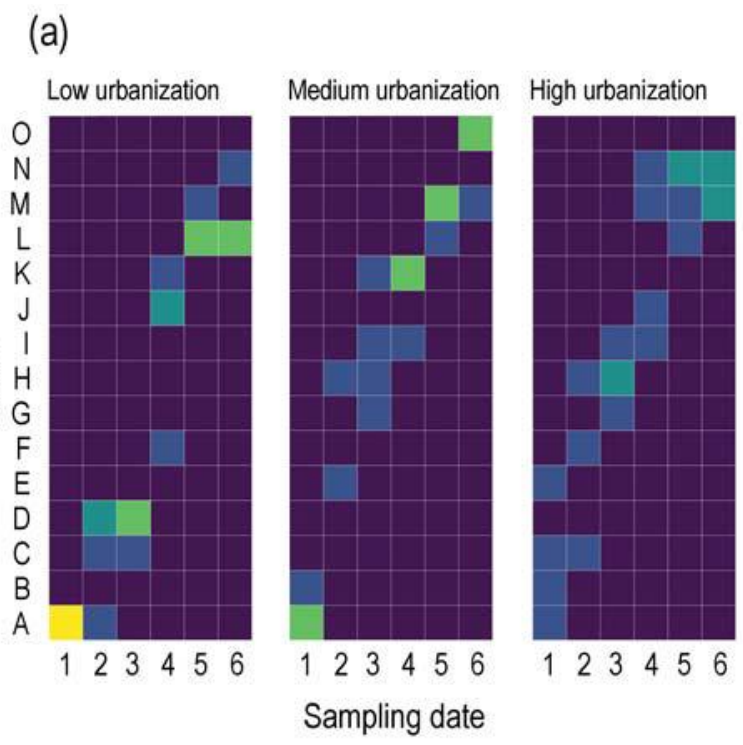

(b)

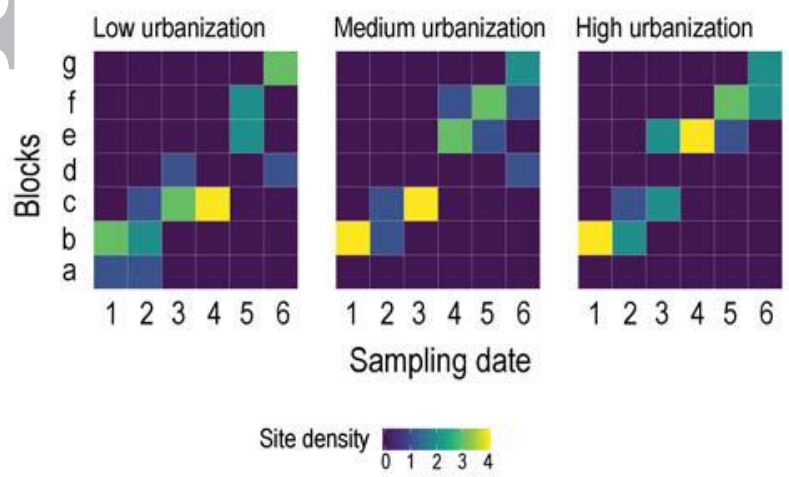

'This article is protected by copyright. All rights reserved.' 
Figure 3. Proportional flower cover (a), pollinator abundance (b) and species richness (c, d) plotted as a function of Julian day of the year for the three urbanization classes (low urbanization: blue squares and line; medium urbanization: yellow triangles and line; high urbanization: orange dots and line). Single points represent one site visit. Solid lines show predicted average values obtained using GLMMs, and shaded areas are 95\% jackknife confidence intervals. Model coefficients and model selection tables for the best models are in Supplementary material Appendix 4 Table A1, A2.
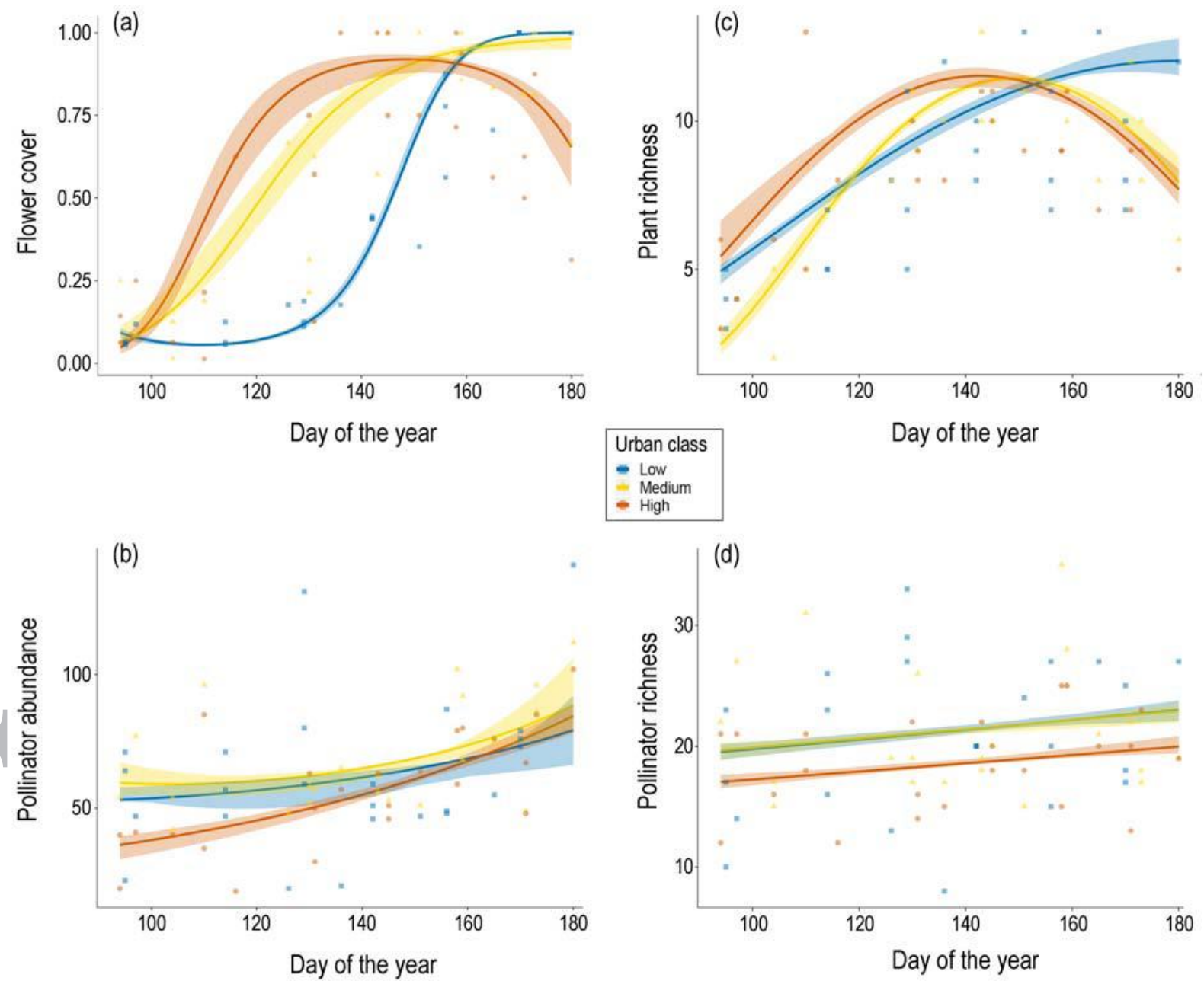


\section{Table Legends}

Table 1. Model selection table for GLMMs for the overall flowering and pollinator phenology. Flower cover was logit transformed, and models had Gaussian family error distribution (identity-link). Models on pollinator abundance had negative binomial distribution (log-link). All models included sites as random factors. Only the best model(s) are shown $(\triangle \mathrm{AICc}<4)$. The complete list of GLMMs coefficient values are reported in Supplementary material Appendix 4 Table A1.

\begin{tabular}{|c|c|c|c|c|c|c|c|c|c|c|c|}
\hline Model & Int & Urb & Day & Day $^{2}$ & Urb:Day & Urb:Day ${ }^{2}$ & $\mathrm{df}$ & $\operatorname{logLik}$ & $\mathrm{AICc}$ & $\triangle \mathrm{AICc}$ & $\mathrm{W}$ \\
\hline \multicolumn{12}{|c|}{ Total flower cover } \\
\hline $\operatorname{Mod} 32$ & + & + & + & + & + & + & 11 & -145.46 & 317.5 & 0.00 & 0.986 \\
\hline \multicolumn{12}{|c|}{ Total pollinator abundance } \\
\hline Mod4 & + & & + & + & & & 5 & -304.71 & 620.4 & 0.00 & 0.246 \\
\hline Mod2 & + & & & + & & & 4 & -305.92 & 620.5 & 0.09 & 0.236 \\
\hline Mod6 & + & + & & + & & & 6 & -304.16 & 621.7 & 1.29 & 0.129 \\
\hline Mod3 & + & & + & & & & 4 & -306.60 & 621.8 & 1.44 & 0.120 \\
\hline Mod8 & + & + & + & + & & & 7 & -303.07 & 622.0 & 1.60 & 0.111 \\
\hline Mod7 & + & + & + & & & & 6 & -304.81 & 623.0 & 2.59 & 0.067 \\
\hline
\end{tabular}

Total flowering plant richness

\begin{tabular}{|c|c|c|c|c|c|c|c|c|c|c|}
\hline Mod4 & + & & + & + & & & 4 & -149.51 & 307.6 & 0.00 \\
\hline Mod24 & + & + & + & + & + & & 8 & -145.32 & 309.0 & 1.40 \\
\hline Mod16 & + & + & + & + & & + & 8 & -145.63 & 309.7 & 2.03 \\
\hline Mod8 & + & + & + & + & & & 6 & -148.17 & 309.7 & 2.06 \\
\hline
\end{tabular}

Total pollinator richness

$\begin{array}{lllllllll}\text { Mod3 } & + & + & & 3 & -227.09 & 460.5 & 0.00 & 0.377 \\ \text { Mod2 } & + & & + & 3 & -227.21 & 460.8 & 0.25 & 0.333 \\ \text { Mod4 } & + & + & + & 4 & -227.01 & 462.6 & 2.10 & 0.132\end{array}$

Int: intercept. Urb: urbanization class (low, medium, high). Day: Julian day of the year.

Day $^{2}$ : squared Julian day of the year to allow for nonlinear effects. W: model weight. +: significant effect of explanatory variables in a given model. 